\title{
HOMOTOPY PERIODICITY OF THE CLASSICAL LIE GROUPS
}

\author{
ALBERT T. LUNDELL
}

The Bott periodicity theorems [4] may be interpreted as follows:

(i) $\pi_{i}(S O(n))=\pi_{i+8}(S O(n+8))$ for $i \leqq n-2$;

(ii) $\pi_{i}(U(n))=\pi_{i+2}(U(n+1))$ for $i \leqq 2 n-1$;

(iii) $\pi_{i}(\boldsymbol{S p}(n))=\pi_{i+8}(\boldsymbol{S p}(n+4))$ for $i \leqq 4 n+1$;

where $S O(n)$ is the special orthogonal group, $U(n)$ is the unitary group, and $\boldsymbol{S p}(n)$ is the symplectic group. Kervaire [9] was able to extend the range of periodicity for $S O(n)$ to read $\pi_{i}(S O(n))$ $=\pi_{i+8}(S O(n+8))$ for $i \leqq n+4$, provided $7 \leqq n$ and $i \not \equiv 6(\bmod 8)$. Some results of Mahowald imply this periodicity for $i \leqq n+4$ and $14 \leqq n$. More recent results of Barratt and Mahowald [2] imply that for suitable exponents $k \geqq 3$ and $18 \leqq i \leqq 2 n-3$,

$$
\pi_{i}(S O(n))=\pi_{i+2 k}\left(S O\left(n+2^{k}\right)\right) .
$$

Notice that according to this result, the homotopy groups of $\operatorname{SO}(n)$ exhibit periodicity of this special type for almost double the stable range. In the case of the unitary group, Kervaire [9] was able to show that $\pi_{2 n+1}(U(n))=\pi_{2(n+2)+1}(U(n+2))$, and Matsunaga [10] has shown that

$$
\pi_{2(n+q)+1}(U(n))=\pi_{2(n+q+24)+1}(U(n+24)) \quad \text { for } q=1,2,
$$

and $n \geqq 3$. It should be noted that all these periodicity theorems for nonstable homotopy groups, with the exception of the results of Barratt and Mahowald, are obtained by explicitly calculating the groups in question, and any periodicity displayed is incidental to the other calculations. For purposes of further calculation of nonstable homotopy groups, it would be useful to know that some appropriately defined periodicity is present, and we will show that for almost double the usual stable range, this is indeed the case.

1. Homotopy periodicity of the Stiefel manifolds. Let $F$ denote the field of real numbers, complex numbers, or quaternions, and $F_{n}$ an $n$-dimensional right vector space over $F$ with the classical inner product. We regard $F_{n}$ as the subspace of $F_{n+1}$ consisting of those vectors with $(n+1)$ th component zero. Let $O_{n}$ be one of the classical Lie groups $S O(n), U(n)$, or $S p(n)$, which operates on $F_{n}$ and preserves

Received by the editors June 22, 1964. 
the inner product. The inclusions $F_{n} \subset F_{n+1}$ induce inclusion homomorphisms $O_{n} \rightarrow O_{n+1}$, and we define $O$ to be the group $\cup_{n=1}^{\infty} O_{n}$, so that $O$ will be one of the groups $S O, U$, or $S p$, depending on the field $F$. Let $O_{n, m}$ be the quotient space $O_{n} / O_{n-m}$, the Stiefel manifold of $m$ frames in $F_{n}$. Then $O_{n, 1}=S_{d n-1}$, the $(d n-1)$-sphere, where $d$ is the dimension of $F$ over the real numbers. We let $V_{n}$ be the quotient space $O / O_{n}$, and point out that there are fibrations $V_{n} \rightarrow V_{n+r}$ with fibre $O_{n+r, r}$.

Let $\lambda_{r}$ be the least in teger such that there is a cross-section of the fibre bundle $O_{\lambda_{r}, r} \rightarrow O_{\lambda_{r}, 1}=S_{d \lambda_{r}-1}$. In the case that $F$ is real, $d=1$, and $\lambda_{r}$ $=2^{\alpha(r)}$, where $\alpha(r)$ is the cardinality of the set $\{s \mid 1 \leqq s \leqq r-1$ and $s \equiv 0,1,2,4(\bmod 8)\}$. In case $F$ is the complex numbers, if $v_{p}(n)$ is the $p$-adic valuation on $n, v_{p}\left(\lambda_{r}\right)=\sup \left\{s+v_{p}(s) \mid 1 \leqq s \leqq(r-1) /(p-1)\right\}$. These results may all be found in [1].

The following theorem is due to James [8, Theorem 3.1], and is the key theorem for homotopy periodicity of the Stiefel manifolds.

TheOREM 1.1. There is a homomorphism

$$
\theta: \pi_{i}\left(O_{n+r, r}\right) \rightarrow \pi_{i+d \lambda_{r}}\left(O_{n+\lambda_{r}+r, r}\right),
$$

which is an epimorphism for $i \leqq 2 d(n+1)-3$, and a monomorphism for $i \leqq 2 d(n+1)-4$.

LEMMA 1.2. The homotopy groups of the space $V_{n}=O / O_{n}$ have the following properties:

(i) $\pi_{i}\left(V_{n}\right)=0$ for $i \leqq d(n+1)-2$;

(ii) $\pi_{d(n+1)-1}\left(V_{n}\right)=Z$ if $d=2,4$, or $n$ is even, $=Z_{2}$ if $d=1$ and $n$ is odd;

(iii) rank $\pi_{d(n+q)-1}\left(V_{n}\right)=1$ for $1 \leqq q$ and $d=2,4$, rank $\pi_{4 q-1}(S O / S O(n))=1$ for $[n / 2] \leqq q,^{1}$ rank $\pi_{2 n}(S O / S O(2 n))=1, \pi_{i}\left(V_{n}\right)$ is a finite group otherwise;

(iv) if $p$ is an odd prime, ${ }^{p} \pi_{i}\left(V_{n}\right)=0^{2}$ for $i \leqq d(n+1)+2 p-5$.

Proof. Parts (i), (ii), and (iii) follow from the fact that in the stable range $\pi_{i}\left(O_{n}\right) \rightarrow \pi_{i}(O)$ is onto, knowledge of $\pi_{i}(O)$ from Bott's theorem [4], knowledge of $\pi_{n-1}(S O(n))$ from Kervaire's paper [9] and knowledge of which of the groups $\pi_{i}\left(O_{n}\right)$ have positive rank (from Serre's $\mathcal{C}$-theory), together with the exact homotopy sequence of the fibration $O_{n} \rightarrow O \rightarrow V_{n}$. Part (iv) follows from the exact homotopy sequence of the fibration $S_{d(n+1)-1} \rightarrow V_{n} \rightarrow V_{n+1}$, and the fact that if $p$ is a prime and $j \leqq 2 p-4,{ }^{p} \pi_{d(n+1)-1+j}\left(S_{d(n+1)-1}\right)=0$.

$1[x]$ denotes the greatest integer in $x$.

2 If $G$ is an abelian group, ${ }^{p} G$ denotes the $p$-primary component of $G$. 
ThEOREM 1.3. There is a homomorphism

$$
\phi: \pi_{i}\left(V_{n}\right) \rightarrow \pi_{i+d \lambda_{r}}\left(V_{n+\lambda_{r}}\right),
$$

which is an epimorphism for $i \leqq 2 d(n+1)-3$, and a monomorphism for $i \leqq 2 d(n+1)-4$, where $r \geqq \max \{1,((i+3) / d)-(n+1)\}$.

Proof. Consider the diagram

$$
\begin{gathered}
\pi_{i+1}\left(V_{n+r}\right) \stackrel{\partial}{\rightarrow} \pi_{i}\left(O_{n+r, r}\right) \stackrel{i_{*}}{\rightarrow} \pi_{i}\left(V_{n}\right) \stackrel{p_{*}}{\rightarrow} \pi_{i}\left(V_{n+r}\right) \\
\pi_{i+j+1}\left(V_{n+\lambda_{r}+r}\right) \stackrel{\partial^{\prime}}{\rightarrow} \pi_{i+j}\left(O_{n+\lambda_{r}+r, r}\right) \stackrel{i_{*}^{\prime}}{\rightarrow} \pi_{i+j}\left(V_{n+\lambda_{r}}\right) \stackrel{p_{*}^{\prime}}{\rightarrow} \pi_{i+j}\left(V_{n+\lambda_{r}+r}\right),
\end{gathered}
$$

where $j=d \lambda_{r}$, the horizontal maps are part of the exact homotopy sequences of the fibrations

$$
O_{n+r, r} \stackrel{i}{\rightarrow} V_{n} \stackrel{p}{\rightarrow} V_{n+r} \text { and } O_{n+\lambda_{r}+r, r} \stackrel{i^{\prime}}{\rightarrow} V_{n+\lambda_{r}} \stackrel{p^{\prime}}{\rightarrow} V_{n+\lambda_{r}+r}
$$

and $\theta$ is the map of 1.1 . Since $r \geqq((i+3) / d)-(n+1)$, we see that $d(n+r+1)-2 \geqq i+1$ and $d\left(n+\lambda_{r}+r+1\right)-2 \geqq i+d \lambda_{r}+1=i+j+1$; so by $1.2 i_{*}$ and $i_{*}^{\prime}$ are isomorphisms. Since $\theta$ is an isomorphism for $i \leqq 2 d(n+1)-4$ and an epimorphism for $i \leqq 2 d(n+1)-3$, the theorem follows if we define $\phi=i_{*}^{\prime} \theta i_{*}^{-1}$.

Theorem 1.4. Let $1 \leqq r, q$. There is a commutative diagram

$$
\begin{array}{ccc}
\pi_{i}\left(O_{n+r, r}\right) \stackrel{i_{*}}{\rightarrow} \pi_{i}\left(V_{n}\right) & \stackrel{p *}{\rightarrow} \pi_{i}\left(V_{n+r}\right) \stackrel{\partial}{\rightarrow} \ldots \\
\downarrow \theta \quad & \downarrow \phi^{\prime} \quad & \downarrow \phi^{\prime} \\
\pi_{j}\left(O_{n+\lambda_{r+q+r, r}}\right) & \stackrel{i_{*}^{\prime}}{\rightarrow} \pi_{j}\left(V_{n+\lambda_{r+q}}\right) \stackrel{p_{*}^{\prime}}{\rightarrow} \pi_{j}\left(V_{n+\lambda_{r+q+r}}\right) \stackrel{\partial^{\prime}}{\rightarrow} \cdots,
\end{array}
$$

where $i \leqq d(n+r+q+1)-3, j \leqq d\left(n+r+q+\lambda_{r+q}+1\right)-3$ and the maps $\phi^{\prime}$ are iterates of the map $\phi$ of 1.3 .

Proof. Consider the commutative diagram

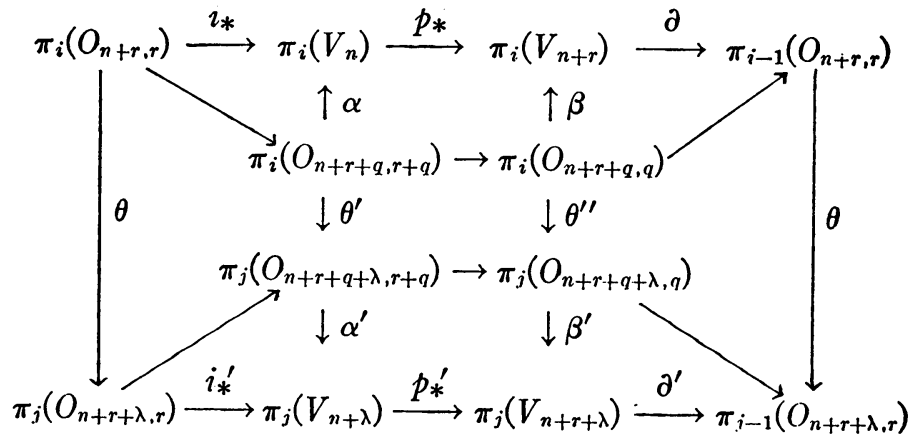


where $j=i+d \lambda_{r+q}$, and $\lambda=\lambda_{r+q}$. The maps $\theta, \theta^{\prime}$, and $\theta^{\prime \prime}$ are the maps of the homotopy sequence of the fibration $O_{n+r+q, r+q} \rightarrow O_{n+r+q, q}$ to the homotopy sequence of the fibration $O_{n+r+q+\lambda, r+q} \rightarrow O_{n+r+q+\lambda, q}$ induced by an element $\theta^{\prime} \in \pi_{d \lambda-1}\left(O_{\lambda, r+q}\right)$, as in James [7, Diagram (2.15)]. Now if $i \leqq d(n+r+q+1)-3$, the maps $\alpha, \beta, \alpha^{\prime}$, and $\beta^{\prime}$ are isomorphisms, and we obtain the theorem if we choose $\phi^{\prime}$ to be the composition of maps which appear in a vertical line in the diagram.

Recall from 1.2 that $\operatorname{rank} \pi_{d(n+r)-1}\left(V_{n}\right)=1$ if $d=2,4$, i.e., if the scalar field is the complex numbers or the quaternions. It is easily shown that the kernel and cokernel of the map

$$
P_{*}: \pi_{d(n+r+1)-1}\left(V_{n}\right) \rightarrow \pi_{d(n+r+1)-1}\left(V_{n+r}\right)
$$

are finite groups, and the cokernel of $p_{*}$ is a cyclic group because $\pi_{d(n+r+1)-1}\left(V_{n+r}\right)=Z$ by 1.2. Let $G_{n, r}$ be the kernel of $p_{*}$ and let $\delta_{n, r}$ be the order of the cokernel of $p_{*}$.

Proposition 1.5. Let $0 \leqq r \leqq n$. Then:

(i) $\delta_{n, r}=\delta_{n+\lambda_{r+1}, r}$,

(ii) $G_{n, r}$ is isomorphic to $G_{n+\lambda_{r+1}, r}$.

Proof. Take $q=1$ in 1.4 so as to obtain the commutative diagram

$$
\begin{aligned}
& \pi_{i}\left(O_{n+r, r}\right) \stackrel{i_{*}}{\rightarrow} \pi_{i}\left(V_{n}\right) \stackrel{p_{*}}{\rightarrow} \pi_{i}\left(V_{n+r}\right) \stackrel{\partial}{\rightarrow} \pi_{i-1}\left(O_{n+r, r}\right) \\
& \downarrow \theta \quad \downarrow \phi^{\prime} \quad \downarrow \phi^{\prime \prime} \quad \downarrow \theta \\
& \pi_{j}\left(O_{n+r+\lambda, r}\right) \stackrel{i_{*}^{\prime}}{\rightarrow} \pi_{j}\left(V_{n+\lambda}\right) \stackrel{p_{*}^{\prime}}{\rightarrow} \pi_{j}\left(V_{n+r+\lambda}\right) \stackrel{\partial^{\prime}}{\rightarrow} \pi_{j-1}\left(O_{n+r+\lambda, r}\right)
\end{aligned}
$$

where $\lambda=\lambda_{r+1}, i=d(n+r+1)-1$, and $j=d(n+r+\lambda+1)-1$. Since $r \leqq n, \phi^{\prime}, \phi^{\prime \prime}$, and $\theta$ are isomorphisms, and it follows that $\phi^{\prime}$ maps $G_{n, r}=$ image $i_{*}$ isomorphically on to $G_{n+\lambda_{r+1}}=$ image $i_{*}^{\prime}$, and that $\theta$ maps cokernel $p_{*}=Z / \delta_{n, r} Z$ isomorphically onto cokernel $p_{*}^{\prime}=Z / \delta_{n+\lambda_{r+1}} Z$, so that $\delta_{n, r}=\delta_{n+\lambda_{r+1}, r}$.

In the complex or quaternionic cases, i.e., $d=2,4$, it is known [5] that

$$
\pi_{d(m+1)-2}\left(O_{m}\right)=Z_{k m}=Z / k_{m} Z, \text { where } k_{m}=c_{m}((d m / 2)-1) !,
$$

and $c_{m}=1$ if $d=2$ or $m$ is odd, $c_{m}=2$ if $d=4$ and $m$ is even.

TheOREM 1.6. If $O_{n}$ is either $U(n)$ or $S p(n)$, and $0 \leqq r$, the sequence

$$
0 \rightarrow G_{n, r} \rightarrow \pi_{d(n+r+1)-2}\left(O_{n}\right) \rightarrow Z_{k_{n+r} / \delta_{n, r}} \rightarrow 0
$$

is exact.

Proof. Consider the commutative diagram 


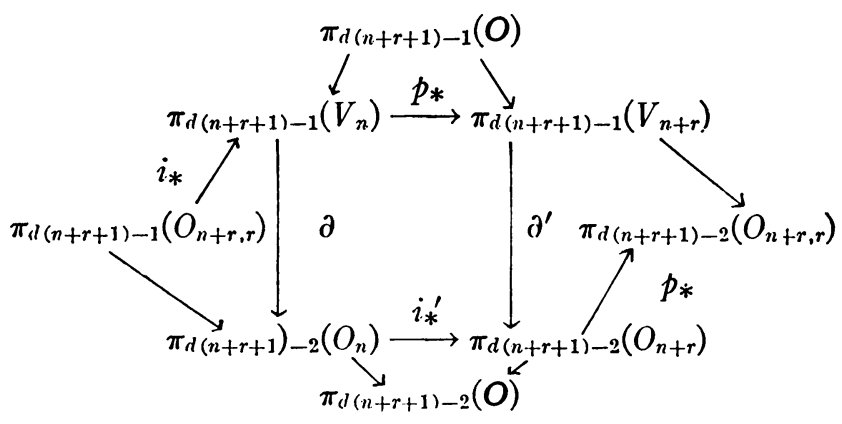

where the horizontal and vertical sequences are exact. By Bott's theorem $\pi_{d(n+r+1)-2}(O)=0$, and $\partial^{\prime}$ is an epimorphism. From the fact that $\pi_{d(n+r+1)-2}\left(O_{n+r}\right)=Z_{k_{n+r}}$, the fact that $\partial^{\prime}$ is an epimorphism, and the definition of $\delta_{n, r}$ it follows that the order of the cokernel of $p_{*}^{\prime}$ is $\delta_{n, r}$, i.e., image $i_{*}^{\prime}=Z_{k_{n+r}} / \delta_{n, r}$. Since $\pi_{d(n+r+1)-1}\left(O_{n+r}\right)$ is finite and $\pi_{d(n+r+1)-1}(O)=Z$, the map $\partial^{\prime}: \pi_{d(n+r+1)}\left(V_{n+r}\right) \rightarrow \pi_{d(n+r+1)-1}\left(O_{n+r}\right)$ is an epimorphism, hence $\partial \mid G_{n, r}$ is an isomorphism. Thus kernel $i_{*}^{\prime}$ is isomorphic to $G_{n, r}$.

2. The unitary group. Specializing to the unitary group, the scalar field is the complex numbers, so we take $d=2$.

Theorem 2.1. Let $1 \leqq r$. There is a homomorphism

$$
\omega: \pi_{2(n+r)-1}(U(n)) \rightarrow \pi_{2\left(n+r+\lambda_{r+1}\right)-1}\left(U\left(n+\lambda_{r+1}\right)\right)
$$

which is an isomorphism if $r \leqq n$.

PRoOF. Recall that $\pi_{2 m}(U)=0, \pi_{2 m-1}(U)=Z$, and $\pi_{2(m+r)-1}(U(m))$ is finite for all $m$ and $1 \leqq r$. Thus if $\Delta$ and $\Delta^{\prime}$ are transgression operators for the fibrations $U \rightarrow U / U(n)$ and $U \rightarrow U / U\left(n+\lambda_{q}\right)$ respectively, they are isomorphisms in the diagram

$$
\begin{gathered}
\pi_{2(n+r)}(U / U(n)) \stackrel{\Delta}{\rightarrow} \pi_{2(n+r)-1}(U(n)) \\
\downarrow \phi \\
\left.\pi_{2\left(n+r+\lambda_{q}\right.}\right)\left(U / U\left(n+\lambda_{q}\right)\right) \stackrel{\Delta^{\prime}}{\rightarrow} \pi_{2\left(n+r+\lambda_{q}\right)-1}\left(U\left(n+\lambda_{q}\right)\right) .
\end{gathered}
$$

Define $\omega$ as the composite $\omega=\Delta^{\prime} \phi \Delta^{-1}$. By $1.3 \phi$ is an isomorphism if $2(n+r) \leqq 4 n$, or $r \leqq n$, where $q \geqq((2 n+2 r+3) / 2)-(n+1)=(2 r+1) / 2$.

The groups $\pi_{2(n+r)}(U(n))$ for $0 \leqq r \leqq n$ do not behave in a periodic manner; for example, $\pi_{2 n}(U(n))=Z_{n !}$. The results of Kervaire [9] and Matsunaga [10], indicate some sort of periodic regularity however, and the following result partially describes this regularity. 
Theorem 2.2. Let $0 \leqq r \leqq n$. There exist finite abelian groups $G_{n, r}$, and integers $\delta_{n, r}$ such that $G_{n, r}$ is isomorphic to $G_{n+\lambda_{r+1}, r}$ and $\delta_{n, r}$ $=\delta_{n+\lambda_{r+1}, r}$, and such that $\pi_{2(n+r)}(U(n))$ is an extension of $G_{n, r}$ by $Z_{(n+r) ! / \delta_{n, r}}$

Proof. The proof is a combination of 1.5 and 1.6 with $O_{n}$ replaced by $U(n)$, and $k_{n+r}=(n+r)$ !.

We have not determined if the group extension is periodic, but we conjecture that it is the trivial extension, and therefore periodic. The groups $G_{n, r}$ and the integers $\delta_{n, r}$ are the subject of work in progress. We may state the following, however, which follows easily from the exact homotopy sequences of the fibrations $S_{2 n-1} \rightarrow V_{n} \rightarrow V_{n+1}$, and knowledge of the $p$-primary components of the homotopy groups of spheres.

Proposition 2.3. If $p$ is an odd prime,

(i) ${ }^{p} G_{n, r}=0$ if $r<(p-1) \inf \{n+1, p\}-1$,

(ii) $v_{p}\left(\delta_{n, r}\right) \leqq[r /(p-1)]$ if $r<p(p-1)$

$$
\leqq[r /(p-1)]+1 \text {, if } p(p-1) \leqq r<2 p(p-1) \text { and } p \leqq n \text {. }
$$

3. The symplectic group. For the symplectic group, the scalar field is the quaternions which have dimension $d=4$ over the real numbers. When it is necessary to distinguish a symbol from the corresponding symbol for the unitary case, we will insert a prime. For example, the in teger $\lambda_{r}^{\prime}$ will be the least integer such that the fibration of quaternionic Stiefel manifolds $O_{\lambda_{r}^{\prime}, r} \rightarrow S_{4 \lambda_{r}^{\prime}-1}$ admits a cross section.

THEOREM 3.1. Let $1 \leqq r$, and let $p$ be an odd prime. There exist homomorphisms

(i) $\omega^{\prime}:{ }^{p} \pi_{4(n+r)-1}(S p(n)) \rightarrow{ }^{p} \pi_{4\left(n+r+\lambda r^{\prime}-1\right.}\left(S p\left(n+\lambda_{r}^{\prime}\right)\right)$,

(ii) $\omega^{\prime}:{ }^{p} \pi_{4(n+r)}(S p(n)) \rightarrow{ }^{p} \pi_{4\left(n+r+\lambda r^{\prime}\right)}\left(S p\left(n+\lambda_{r}^{\prime}\right)\right)$,

(iii) $\omega^{\prime}:{ }^{p} \pi_{4(n+r)+1}(S p(n)) \rightarrow{ }^{p} \pi_{4\left(n+r+\lambda_{r+1)+1}^{\prime}\right.}\left(S p\left(n+\lambda_{r+1}\right)\right)$.

If $n+r$ is even, each of these maps $\omega^{\prime}$ is also a homomorphism of the 2-primary component. Moreover, the first map is an isomorphism for $r \leqq n+1$, and the other two are isomorphisms for $i \leqq n$.

Proof. The proof is essentially the same as the proof of 2.1, and we will omit it. We restrict our attention to the $p$-primary component for an odd prime $p$, because the groups $\pi_{4(n+r)}(S p)=Z_{2}$ $=\pi_{4(n+r)+1}(S p)$ for $n+r$ odd.

THEOREM 3.2. Let $0 \leqq r \leqq n$. There exist finite abelian groups $G_{n, r}^{\prime}$, and integers $\delta_{n, r}^{\prime}$ such that $G_{n, r}^{\prime}$ is isomorphic to $G_{n+\lambda_{r+1}^{\prime}, r}, \delta_{n, r}^{\prime}=\delta_{n+\lambda_{r+1}, r}^{\prime}$, and such that $\pi_{4(n+r)+2}(S p(n))$ is an extension of $G_{n, r}^{\prime}$ by $Z_{b_{n+r}(2(n+r)-1) ! / \delta^{\prime}{ }_{n, r},}$ where $b_{n+r}=2$ if $n+r$ is even and $b_{n+r}=1$ if $n+r$ is odd. 
Proof. The proof is a combination of 1.5 and 1.6 with $O_{n}$ replaced by $S_{p}(n)$, and $k_{n+r}=b_{n+r}(2(n+r)-1)$ !.

As in the unitary case, we conjecture that the extension is trivial but have not proved this. The proof of the following proposition is similar to the proof of 2.3 .

Proposition 3.3. If $p$ is an odd prime,

(i) ${ }^{p} G_{n, r}^{\prime}=0$ if $r<p(p-1) / 2$ or $r<p(p-1) \leqq p n$;

(ii) $v_{p}\left(\delta_{n, r}^{\prime}\right) \leqq[2 r /(p-1)]$ if $r<p(p-1) / 2$

$$
\leqq[2 r /(p-1)]+1 \text { if } p(p-1) / 2 \leqq r<p(p-1) \leqq n(p-1) \text {; }
$$

(iii) ${ }^{p} \pi_{4(n+r)-1}(S p(n))=0$ if $r<p(p-1) / 2$ or $r<(p+1)(p-1) / 2$ and $p-1 \leqq n$;

(iv) ${ }^{p} \pi_{4(n+r)}(\boldsymbol{S p}(n))=0$ if $r<((p-1) / 2) \inf \{2 n+2, p\}$;

(v) ${ }^{p} \pi_{4(n+r)+1}(\operatorname{Sp}(n))=0$ if $r<(p-1) / 2$.

4. The special orthogonal group. The main theorem on periodicity in the homotopy groups of the special orthogonal groups is based on the Bott periodicity theorems and the following result due to Barratt and Mahowald which will be stated without proof.

Proposition 4.1 (Barratt and Mahowald). If $4<n$, the homomorphism $i_{*}: \pi_{4 n-1}(S O(2 n+1)) \rightarrow \pi_{4 n-1}(S O)$ is an epimorphism.

Proposition 4.2. For $18 \leqq i \leqq 4[(n-1) / 2]+1$

$$
\pi_{i}(S O(n))=\pi_{i+1}(S O / S O(n)) \oplus \pi_{i}(S O) \quad \text { (direct sum). }
$$

PROof. Let $\epsilon_{m}$ be a generator of $\pi_{m}(S O)$, let $\eta_{m}$ generate $\pi_{m+1}\left(S_{m}\right)$, and let $\theta_{m}$ generate $\pi_{m+2}\left(S_{m}\right), 3 \leqq m$. Kervaire [9] has shown that $\epsilon_{8 n}=\epsilon_{8 n-1} \circ \eta_{8 n-1}$, and $\epsilon_{8 n+1}=\epsilon_{8 n-1} \circ \theta_{8 n-1}$. Since $\pi_{4 n-1}(S O)=Z$, there is a splitting homomorphism $\beta: \pi_{4 n-1}(S O) \rightarrow \pi_{4 n-1}(S O(2 n+1))$ for $4<n$. Since $\eta_{8 n-1}$ and $\theta_{8 n-1}$ are suspensions we may define splitting homomorphisms $\beta^{\prime}: \pi_{8 n}(\mathrm{SO}) \rightarrow \pi_{8 n}(S O(4 n+1))$ and $\beta^{\prime \prime}: \pi_{8 n+1}(\mathrm{SO})$ $\rightarrow \pi_{8 n+1}(S O(4 n+1))$ by $\beta^{\prime}\left(\epsilon_{8 n}\right)=\beta\left(\epsilon_{8 n-1}\right) \circ \eta_{8 n-1}$, and $\beta^{\prime \prime}\left(\epsilon_{8 n+1}\right)$ $=\beta\left(\epsilon_{8 n-1}\right) \circ \theta_{8 n-1}$, if $n>2$. The splitting is trivial in the other cases because $\pi_{i}(S O)=0$ for $i \not \equiv 0,1,3,7(\bmod 8)$.

Combining this with the Bott theorem [4] that $\pi_{i}(S O)=\pi_{i+8}(S O)$, and 1.3, we obtain the following periodicity theorem.

Theorem 4.3. If $20-n \leqq r \leqq n-1$,

$$
\pi_{n-2+r}(S O(n))=\pi_{n+2 \sigma r-2+r}\left(S O\left(n+2^{\sigma_{r}}\right)\right),
$$

where $\sigma_{r}=\sup \{3, \alpha(r)\} .^{3}$

Observe that for $r=0$ this theorem reduces to the usual Bott

$\alpha(r)$ is defined in $\$ 1$. 
periodicity, but periodicity persists as far as $\pi_{2 n-3}(S O(n))$, or about twice the stable range. Also observe that the further from the stable range a group is, the longer the period. This is also true of the periodicity for $U(n)$ and $S p(n)$.

If $p$ is an odd prime, we may show that the $p$-primary components of $\pi_{i}(S O(n))$ behave with periodic regularity for about four times the stable range. To see this one uses the fact, due to Harris [6], that $\pi_{i}(S O(2 n+1))$ is $\mathcal{C}$ isomorphic to $\pi_{i}(S p(n))$, where $\mathcal{C}$ is the class of 2-primary groups. Combining this with 3.1 and 3.2, one obtains the following theorem.

Theorem 4.4. Let $p$ be an odd prime, and let $0 \leqq r=i+1-n$. Then

(i) ${ }^{p} \pi_{4 i-1}(S O(2 n-1))={ }^{p} \pi_{4(i+\lambda r)-1}\left(S O\left(2 n+2 \lambda_{r}^{\prime}-1\right)\right)$ if $i \leqq 2 n-1$;

(ii) ${ }^{p} \pi_{4 i}(S O(2 n-1))={ }^{p} \pi_{4(i+\lambda r)}\left(S O\left(2 n+2 \lambda_{r}^{\prime}-1\right)\right)$ if $i \leqq 2 n-2$;

(iii) ${ }^{p} \pi_{4 i+1}(S O(2 n-1))={ }^{p} \pi_{4\left(i+\lambda_{r+1}+1\right.}^{\prime}\left(S O\left(2 n+2 \lambda_{r+1}^{\prime}-1\right)\right)$ if $i \leqq 2 n$ -2 ;

(iv) ${ }^{p} \pi_{4 i+2}(S O(2 n-1))$ is an extension of ${ }^{p} G_{n-1, r}^{\prime}$ by the group ${ }^{p} Z_{b_{i}(2 i-1) ! / \delta^{\prime}{ }_{n-1, r}}$ if $i \leqq 2 n-2$.

To obtain periodicity theorems for the groups ${ }^{p} \pi_{i}(S O(2 n))$, one uses the result of Borel and Serre [3] that for an odd prime $p$, ${ }^{p} \pi_{i}(S O(2 n))={ }^{p} \pi_{i}\left(S_{2 n-1}\right) \oplus^{p} \pi_{i}(S O(2 n-1))$ (direct sum), and the fact that the $(2 m)$-fold suspension $E^{2 m}:{ }^{p} \pi_{i}\left(S_{2 n-1}\right) \rightarrow^{p} \pi_{i+2 n}\left(S_{2(m+n)-1}\right)$ is an isomorphism onto for $i \leqq p n-4$, together with 4.4. Roughly, ${ }^{p} \pi_{i}(S O(2 n))$ exhibits periodic regularity for $i \leqq \inf \{8 n-8, p n-4\}$.

\section{BIBLIOGRAPHY}

1. J. F. Adams, Applications of the Grothendieck-Atiyah-Hirzebruch functor $K(X)$, pp. 104-113. Colloquium on Algebraic Topology, Aarhus Univ., 1962.

2. M. G. Barratt and M. Mahowald, The metastable homotopy of $O(n)$, Bull. Amer. Math. Soc. 70 (1964), 758-760.

3. A. Borel and J. P. Serre, Groupes de Lie et puissances reduites de Steenrod, Amer. J. Math. 73 (1953), 409-448.

4. R. Bott, The stable homotopy of the classical groups, Ann. of Math. 70 (1959), 313-337.

5. - A note on the Samelson product in the classical groups, Comment. Math. Helv. 34 (1960), 249-256.

6. B. Harris, On the homotopy groups of the classical groups, Ann. of Math. 74 (1961), 407-413.

7. I. M. James, The intrinsic join, Proc. London Math. Soc. 8 (1958), 507-535.

8. - Cross-sections of Stiefel manifolds, Proc. London Math. Soc. 8 (1958), 536-547.

9. M. Kervaire, Some nonstable homotopy groups of Lie groups, Illinois J. Math. 4 (1960), 161-169.

10. H. Matsunaga, The homotopy groups $\pi_{2 n+i}(U(n))$ for $i=3,4$, and 5 , Mem. Fac. Sci. Kyushu Univ. Ser. A 15 (1961), 72-81.

UNIVERSITY OF CoLORADO 